\title{
Noise influence on solid-liquid transition of ultrathin lubricant film
}

\author{
Alexei V. Khomenko \\ Physical Electronics Department, Sumy State University, Rimskii-Korsakov St. 2, 40007 Sumy, Ukraine \\ Received 5 March 2004; received in revised form 3 June 2004; accepted 30 June 2004 \\ Available online 10 July 2004 \\ Communicated by R. Wu
}

\begin{abstract}
The melting of ultrathin lubricant film by friction between atomically flat surfaces is studied. The additive noises of the elastic shear stress and strain, and the temperature are introduced for building the phase diagrams with the domains of sliding, stick-slip, and dry friction. It is shown that increase of the strain noise intensity causes the lubricant film melting even at low temperatures of the friction surfaces.
\end{abstract}

(C) 2004 Elsevier B.V. All rights reserved.

PACS: 64.60.-i; 05.10.Gg; 62.20.Qp; 68.60.-p

Keywords: Viscoelastic medium; Melting; Stick-slip friction

\section{Introduction}

The study of the noise influence on the friction process has an evident fundamental and practical importance because in some experimental situations the fluctuations can change the frictional behavior critically, for example, providing the conditions for low friction [1-3]. In particular, the thermal noise, acting in any experiments, can convert the ultrathin lubricant film from stable solidlike phase state to the liquidlike one and, thus, transform the dry friction into the sliding or the stick-slip (the interrupted) modes. Therefore, in recent years the considerable study has been given to the influence of disorder and random impurities in the interface on the static and the dynamic frictional phenomena [4-6]. These investigations show that a periodic surfaces are characterized by smaller friction coefficient during sliding than non-regular ones. Besides, the stick-slip dynamics, inherent in solid friction, attracts an increased attention on the atomic [7-9] and the macroscopic [10,11] levels as well as for granular mediums [1214]. In order to achieve the better understanding of the above phenomena, here an analytic approach is put forward, which describes the transitions between friction modes due to variation of fluctuations of elastic and thermal fields.

E-mail address: khom@phe.sumdu.edu.ua (A.V. Khomenko). 
In the previous work [15] on the basis of rheological description of viscoelastic medium the system of kinetic equations has been obtained, which define the mutually coordinated evolution of the elastic shear components of the stress $\sigma$ and the strain $\varepsilon$, and the temperature $T$ in ultrathin lubricant film during friction between atomically flat mica surfaces. Let us write these equations using the measure units

$$
\sigma_{s}=\left(\frac{\rho c_{v} \eta_{0} T_{c}}{\tau_{T}}\right)^{1 / 2}, \quad \varepsilon_{s}=\frac{\sigma_{s}}{G_{0}} \equiv\left(\frac{\tau_{\varepsilon}}{\tau_{T}}\right)^{1 / 2}\left(\frac{\rho c_{v} T_{c} \tau_{\varepsilon}}{\eta_{0}}\right)^{1 / 2}, \quad T_{c}
$$

for variables $\sigma, \varepsilon, T$, respectively, where $\rho$ is the mass density, $c_{v}$ is the specific heat capacity, $T_{c}$ is the critical temperature, $\eta_{0} \equiv \eta\left(T=2 T_{c}\right)$ is the typical value of shear viscosity $\eta, \tau_{T} \equiv \rho l^{2} c_{v} / \kappa$ is the time of heat conductivity, $l$ is the scale of heat conductivity, $\kappa$ is the heat conductivity constant, $\tau_{\varepsilon}$ is the relaxation time of matter strain, $G_{0} \equiv \eta_{0} / \tau_{\varepsilon}$ :

$$
\begin{aligned}
& \tau_{\sigma} \dot{\sigma}=-\sigma+g \varepsilon, \\
& \tau_{\varepsilon} \dot{\varepsilon}=-\varepsilon+(T-1) \sigma, \\
& \tau_{T} \dot{T}=\left(T_{e}-T\right)-\sigma \varepsilon+\sigma^{2} .
\end{aligned}
$$

Here the stress relaxation time $\tau_{\sigma}$, the temperature $T_{e}$ of atomically flat mica friction surfaces, and the constant $g=G / G_{0}$ are introduced, where $G$ is the lubricant shear modulus. It can be seen $[15,16]$ that Eqs. (2) and (3) are the Maxwell-type and the Kelvin-Voigt equations for viscoelastic matter, correspondingly. The latter takes into account the dependence of the shear viscosity on the dimensionless temperature $\eta=\eta_{0} /(T-1)$. Eq. (4) represents the heat conductivity expression, which describes the heat transfer from the friction surfaces to the layer of lubricant, the effect of the dissipative heating of a viscous liquid flowing under the action of the stress, and the reversible mechanic-and-caloric effect in linear approximation. These equations coincide with the synergetic Lorenz system formally $[17,18]$, where the elastic shear stress acts as the order parameter, the conjugate field is reduced to the elastic shear strain, and the temperature is the control parameter. As is known this system can be used for description of the thermodynamic phase and the kinetic transitions.

In Ref. [15] a melting of ultrathin lubricant film by friction between atomically flat mica surfaces has been represented as a result of action of spontaneously appearing elastic field of stress shear component caused by the heating of friction surfaces above the critical value $T_{c}=1+g^{-1}$. Thus, according to such approach the studied solid-liquid transition of lubricant film occurs due to both thermodynamic and shear melting. The initial reason for this self-organization process is the positive feedback of $T$ and $\sigma$ on $\varepsilon$ (see Eq. (3)) conditioned by the temperature dependence of the shear viscosity leading to its divergence. On the other hand, the negative feedback of $\sigma$ and $\varepsilon$ on $T$ in Eq. (4) plays an important role since it ensures the system stability.

According to this approach the lubricant represents a strongly viscous liquid that can behave itself similar to the solid - has a high effective viscosity and still exhibits a yield stress [7,16]. Its solidlike state corresponds to the elastic shear stress $\sigma=0$ because the Maxwell-type equation (2), describing the elastic properties at steady state $\dot{\sigma}=0$, falls out of consideration. Eq. (3), containing the viscous stress, reduces to the Debye law describing the rapid relaxation of the elastic shear strain during the microscopic time $\tau_{\varepsilon} \approx a / c \sim 10^{-12} \mathrm{~s}$, where $a \sim 1 \mathrm{~nm}$ is the lattice constant or the intermolecular distance and $c \sim 10^{3} \mathrm{~m} / \mathrm{s}$ is the sound velocity. At that the heat conductivity equation (4) takes the form of simplest expression for temperature relaxation that does not contain the terms representing the dissipative heating and the mechanic-and-caloric effect of a viscous liquid. At non-zero value of $\sigma$ Eqs. (2)-(4) describes the above mentioned properties inherent in the liquidlike state of lubricant.

Moreover, in accordance with Ref. [11] in the absence of shear deformations the temperature mean-square displacement is defined by equality $\left\langle u^{2}\right\rangle=T / G a$. The average shear displacement is found from the relationship $\left\langle u^{2}\right\rangle=\sigma^{2} a^{2} / G^{2}$. The total mean-square displacement represents the sum of these expressions provided that the thermal fluctuations and the stress are independent. Above implies that the transition of lubricant from solidlike to fluidlike state is induced both by heating and under influence of stress generated by solid surfaces at friction. This agrees with examination of solid state instability within the framework of shear and dynamic disorder-driven 
melting representation in absence of thermal fluctuations. Thus, the strain fluctuations, related to the stress ones, and the thermal fluctuations will be considered independently. It is assumed that the film becomes more liquidlike and the friction force decreases with the temperature growth due to decreasing activation energy barrier to molecular hops. Besides, the friction force decreases with increasing velocity at the contact $V=l \partial \varepsilon / \partial t$ because the latter leads to the growth of the shear stress $\sigma$ according to the Maxwell stress-strain $\varepsilon$ relation: $\partial \sigma / \partial t=-\sigma / \tau_{\sigma}+$ $G \partial \varepsilon / \partial t$.

In present Letter the additive noises of the shear components of the elastic stress and strain, and the temperature are taken into account in a lubricant film. The solidlike lubricant is assumed to be amorphous (disordered). Therefore I study the glass transition represented in terms of a second-order phase transition. It is shown that increase of the strain noise intensity causes the lubricant film melting even at low temperatures of friction surfaces, at which the temperature noise plays a crucial role. The phase diagrams are calculated defining the domains of sliding, stickslip, and dry friction in the planes temperature noise intensity - temperature of friction surfaces and noise intensity of shear elastic strain-temperature noise intensity.

\section{Langevin and Fokker-Planck equations}

Consider now the affect of additive noises of the elastic stress and strain shear components $\sigma, \varepsilon$, and the temperature $T$. With this aim, one should add to right-hand sides of Eqs. (2)-(4) the stochastic terms $I_{\sigma}^{1 / 2} \xi, I_{\varepsilon}^{1 / 2} \xi$, $I_{T}^{1 / 2} \xi$ (here the noise intensities $I_{\sigma}, I_{\varepsilon}, I_{T}$ are measured in units of $\sigma_{s}^{2}, \varepsilon_{s}^{2} \tau_{\varepsilon}^{-2},\left(T_{c} \kappa / l\right)^{2}$, correspondingly, and $\xi(t)$ is the $\delta$-correlated stochastic function) [19]. Experimental data for organic lubricant [7] show that relaxation time of the stress $\tau_{\sigma}$ at normal pressure is $\sim 10^{-10} \mathrm{~s}$, and it increases by several orders of magnitude at large pressures. Since the ultrathin lubricant film consists of less than ten molecular layers the relaxation process of the temperature to the value $T_{e}$ occurs during time satisfying the condition $\tau_{T} \ll \tau_{\sigma}$. Then, within the adiabatic approximation $\tau_{\sigma} \gg \tau_{\varepsilon}, \tau_{T}$, Eqs. (3) and (4) are reduced to the time dependencies

$$
\begin{aligned}
& \varepsilon(t)=\bar{\varepsilon}+\tilde{\varepsilon} \xi(t), \quad T(t)=\bar{T}+\tilde{T} \xi(t), \\
& \bar{\varepsilon} \equiv \sigma\left(T_{e}-1+\sigma^{2}\right) d(\sigma), \quad \tilde{\varepsilon} \equiv \sqrt{I_{\varepsilon}+I_{T} \sigma^{2}} d(\sigma), \\
& \bar{T} \equiv\left(T_{e}+2 \sigma^{2}\right) d(\sigma), \quad \tilde{T} \equiv \sqrt{I_{T}+I_{\varepsilon} \sigma^{2}} d(\sigma), \quad d(\sigma) \equiv\left(1+\sigma^{2}\right)^{-1} .
\end{aligned}
$$

Here, deterministic components are reduced to obtained in Ref. [15], whereas fluctuational ones follow from the known property of variance additivity of independent Gaussian random quantities [19]. Thus, using the slaving principle inherent in synergetics $[17,18]$ transforms noises of both strain $\varepsilon$ and temperature $T$, which are adiabatic initially, to multiplicative form. As a result, a combination of Eqs. (2), (5), and (6) leads to the Langevin equation

$$
\tau_{\sigma} \dot{\sigma}=f(\sigma)+\sqrt{I(\sigma)} \xi(t), \quad f \equiv-\frac{\partial V}{\partial \sigma},
$$

where the force $f$ is related to the synergetic potential [15]

$$
V=\frac{1}{2}(1-g) \sigma^{2}+g\left(1-\frac{T_{e}}{2}\right) \ln \left(1+\sigma^{2}\right)
$$

and an expression for the effective noise intensity

$$
I(\sigma) \equiv I_{\sigma}+\left(I_{\varepsilon}+I_{T} \sigma^{2}\right) g^{2} d^{2}(\sigma)
$$

is obtained in accordance with above mentioned property of noise variance additivity. In order to avoid mistakes, one should notice that a direct insertion of Eqs. (5) and (6) into (2) results in the appearance of a stochastic addition

$$
\left[I_{\sigma}^{1 / 2}+\left(I_{\varepsilon}^{1 / 2}+I_{T}^{1 / 2} \sigma\right) g d(\sigma)\right] \xi(t)
$$


whose squared amplitude is quite different from the effective noise intensity (9). Moreover, in contrast to the expressions (6), a direct use of the adiabatic approximation in Eqs. (3) and (4) reduces the fluctuational additions in Eq. (5) to the forms: $\tilde{\varepsilon} \equiv\left(I_{\varepsilon}^{1 / 2}+I_{T}^{1 / 2} \sigma\right) d(\sigma), \tilde{T} \equiv\left(I_{T}^{1 / 2}-I_{\varepsilon}^{1 / 2} \sigma\right) d(\sigma)$. The latter is obviously erroneous since the effective noise of the temperature $\tilde{T}$ disappears entirely for the stress $\sigma=\sqrt{I_{T} / I_{\varepsilon}}$. The reason for such a contradiction is that the Langevin equation does not permit the use of usual analysis methods (see [19]).

To continue in the usual way, let us write the Fokker-Planck equation related to the Langevin equation (7):

$$
\frac{\partial P(\sigma, t)}{\partial t}=\frac{\partial}{\partial \sigma}\left\{-f(\sigma) P(\sigma, t)+\frac{\partial}{\partial \sigma}[I(\sigma) P(\sigma, t)]\right\} .
$$

At steady state, that is the single considered case, the probability distribution $P(\sigma, t)$ becomes a time-independent function $P(\sigma)$. Consequently, under the usual condition, that the expression in braces of the right-hand side of Eq. (11) is equal to zero, this leads to a stationary distribution

$$
P(\sigma)=Z^{-1} \exp \{-U(\sigma)\},
$$

where $Z$ is a normalization constant. The effective potential

$$
U(\sigma)=\ln I(\sigma)-\int_{0}^{\sigma} \frac{f\left(\sigma^{\prime}\right)}{I\left(\sigma^{\prime}\right)} \mathrm{d} \sigma^{\prime}, \quad f \equiv-\frac{\partial V}{\partial \sigma},
$$

is determined by the synergetic potential $V$ (Eq. (8)) and the noise intensity $I(\sigma)$ (Eq. (9)) [20]. Combining these expressions, one can find the explicit form of $U(\sigma)$, which is too cumbersome to be reproduced here. The equation defining the locations of the maximums of the distribution function $P(\sigma)$

$$
(1-g) x^{3}+g\left(2-T_{e}\right) x^{2}-2 g^{2} I_{T} x+4 g^{2}\left(I_{T}-I_{\varepsilon}\right)=0, \quad x \equiv 1+\sigma^{2},
$$

is much simpler. According to Eq. (14) maximums are insensitive to changes in the intensity of the noise $I_{\sigma}$ of the stress $\sigma$, but they are determined by the value $T_{e}$ of the friction surfaces temperature and the intensities $I_{\varepsilon}$ and $I_{T}$ of the noises of the strain $\varepsilon$ and the lubricant film temperature $T$, which acquire the multiplicative character in Eq. (9). Hence, for simplicity $I_{\sigma}$ can be set equal to 0 and Eqs. (8), (9), and (13) give the following expression for the effective potential:

$$
\begin{aligned}
U(\sigma)= & \frac{1}{2 g^{2} I_{T}}\left\{i\left[i(1-g)-g\left(2-T_{e}\right)\right] \ln \left|1+\frac{\sigma}{i}\right|+(1-g) \frac{\sigma^{2}}{2}+\left[g\left(2-T_{e}\right)-i(1-g)\right] \sigma\right\} \\
& +\ln \left[g^{2} d^{2}(\sigma)\left(I_{\varepsilon}+\sigma^{2} I_{T}\right)\right], \quad i \equiv \frac{I_{\varepsilon}}{I_{T}}-1 .
\end{aligned}
$$

\section{Phase diagrams}

According to Eq. (14) the effective potential (15) has a minimum at $\sigma=0$ if the temperature $T_{e}$ does not exceed the critical level

$$
T_{c}=1+g^{-1}+2 g\left(I_{T}-2 I_{\varepsilon}\right)
$$

whose value increases at increasing the characteristic value of shear viscosity $\eta_{0}$ and the temperature noise intensity, but decreases with growth of the shear modulus $G$ of lubricant and the strain noise. Here, the lubricant film does not melt. The solutions of Eq. (14)

$$
\sigma_{ \pm}^{2}=\frac{1}{2}\left[\frac{g\left(T_{e}-2\right)}{1-g}-3 \pm \sqrt{\left(\frac{g\left(T_{e}-2\right)}{1-g}+7\right)\left(\frac{g\left(T_{e}-2\right)}{1-g}-1\right)}\right]
$$


are obtained on the line defined by expression (16) after elimination of the root $\sigma^{2}=0$. At $T_{e}<T_{c}^{0}=2\left(1+2 g^{-1}\right) / 3$ the roots $\sigma_{ \pm}$are complex, starting from $T_{e}=T_{c}^{0}$ they become zero, and at $T_{e}>T_{c}^{0}$ one has real magnitudes $\sigma_{+}=-\sigma_{-} \neq 0$ that implies lubricant film melting. If equality (16) is fulfilled, the root $\sigma=0$ corresponds to the minimum of the effective potential (15) at $T_{e}<T_{c}^{0}$, whereas at $T_{e}>T_{c}^{0}$ this root corresponds to the maximum, and the roots $\sigma_{ \pm}$- to symmetrical minimums.

Now, let us find another condition for the stability of the roots $\sigma_{ \pm}$in the simple case $I_{\varepsilon}=0$. Setting the discriminant of Eq. (14) equal to zero, one gets the equations

$$
I_{T}=0, \quad I_{T}^{2}-I_{T}\left\{\frac{27}{2 g}\left[\frac{1-g}{g}+\frac{2-T_{e}}{3}\right]-\frac{\left(2-T_{e}\right)^{2}}{8(1-g)}\right\}-\frac{\left(2-T_{e}\right)^{3}}{2 g(1-g)}=0,
$$

the second of which gives

$$
2 I_{T}=\frac{27}{2 g}\left[\frac{1-g}{g}+\frac{2-T_{e}}{3}\right]-\frac{\left(2-T_{e}\right)^{2}}{8(1-g)} \pm\left\{\left[\frac{27}{2 g}\left(\frac{1-g}{g}+\frac{2-T_{e}}{3}\right)-\frac{\left(2-T_{e}\right)^{2}}{8(1-g)}\right]^{2}+\frac{2\left(2-T_{e}\right)^{3}}{g(1-g)}\right\}^{1 / 2} .
$$

This equation defines a bell-shaped curve $T_{e}\left(I_{T}\right)$, which intersects the horizontal axis at the point

$$
I_{T}=\frac{9(3-4 g)+8 g^{2}}{2 g^{2}(1-g)}+\left\{\left[\frac{9(3-4 g)+8 g^{2}}{2 g^{2}(1-g)}\right]^{2}+\frac{16}{g(1-g)}\right\}^{1 / 2}
$$

and vertical axis at the point $T_{e}=2$. It has a maximum $T_{e}=2 g^{-1}$ at

$$
I_{T}=\frac{2(1-g)}{g^{2}} .
$$

It is easy to see that line (16) touches the curve (19) at the tricritical point

$$
T_{e}=T_{c}^{0}=\frac{2}{3}\left(1+2 g^{-1}\right), \quad I_{T}=\frac{1-g}{6 g^{2}} .
$$

Thus, this point addresses to the appearance of real roots $\sigma_{ \pm} \neq 0$ (17) of Eq. (14) that means lubricant film melting.

Let us now consider the more general case of two multiplicative noises $I_{\varepsilon}, I_{T} \neq 0$. The condition of extremum of the effective potential (15) splits into two equations, one of which is simply $\sigma=0$, and the other one is given by Eq. (14). As mentioned above, the analysis of the latter indicates that the line of existence of the zero solution is defined by expression (16). The tricritical point $T$ has the coordinates

$$
T_{e}=\frac{2}{3}\left(1+2 g^{-1}-2 g I_{\varepsilon}\right), \quad I_{T}=\frac{1}{6 g}\left(g^{-1}-1+8 g I_{\varepsilon}\right) .
$$

The phase diagrams for the fixed intensities $I_{\varepsilon}$ are shown in Fig. 1. Physically, one should take into consideration that lines 1 and 2 define the thresholds of stability loss of the system, i.e., boundaries of domains corresponding to the different forms of effective potential (15). Above straight line 1 the potential (15) has a minimum only at nonzero stress $\sigma$ and the system manifests a stable sliding friction (SF) inherent in the liquidlike phase of lubricant. Below curve 2 the potential (15) has a minimum only at $\sigma=0$ and the dry friction (DF) occurs that is characteristic for the solidlike state of lubricant film. Between these lines the potential (15) has minimums at zero and non-zero $\sigma$ and the region of the stick-slip friction (SS) mode is realized, i.e., mode that is characterized by periodic transitions between two dynamic states during steady-state sliding. It is relevant to an intermittent regime of lubricant melting, where a mixture of both solidlike and liquidlike phases exists. For $I_{\varepsilon}<\left(1+g^{-1}\right) / 4 g$ the situation is generally the same as in the simple case $I_{\varepsilon}=0$ (see Fig. 1a). At $I_{\varepsilon}>\left(1+g^{-1}\right) / 4 g$ the sliding friction is possible even for small values of temperature $T_{e}$ of friction surfaces and noise intensities $I_{T}$ of the lubricant film temperature (Fig. 1b). According to (23) the tricritical point lies on the $I_{T}$-axis at $I_{\varepsilon}=\left(1+2 g^{-1}\right) / 2 g$, and if the noise intensity $I_{\varepsilon}$ is larger than the critical value $I_{\varepsilon}=2 g^{-2}$, the stable dry friction domain disappears (see Fig. 1c). It is worth noting 


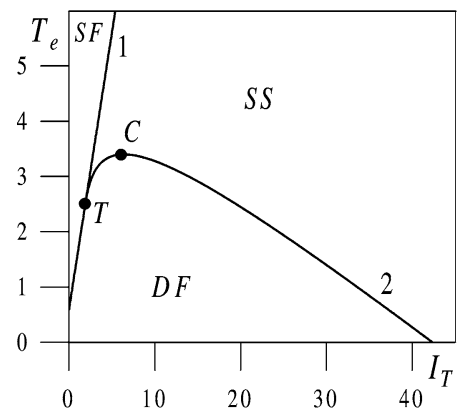

b
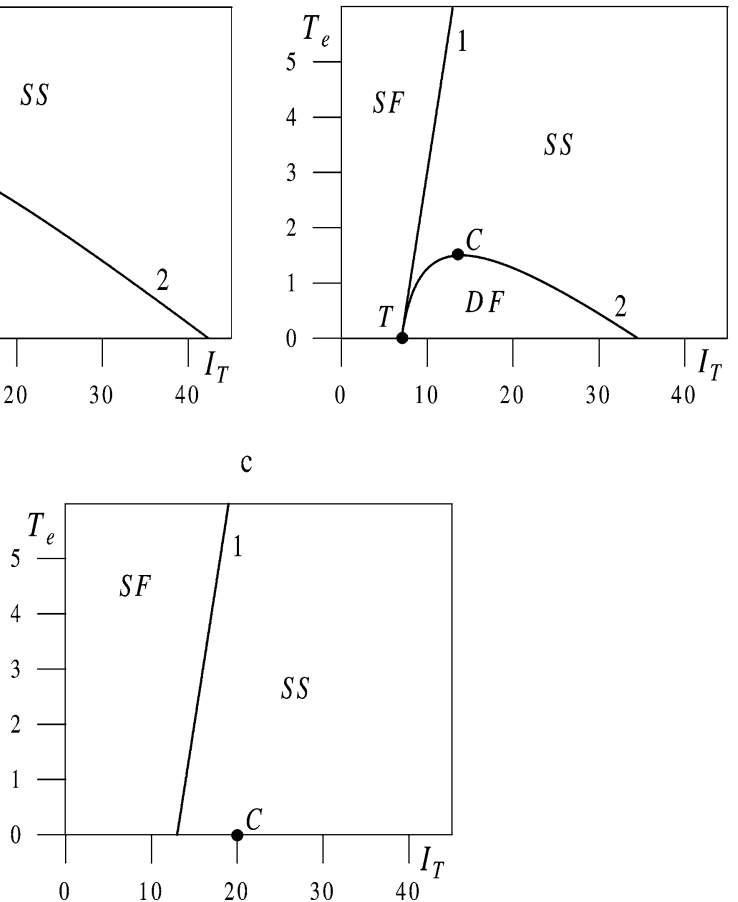

Fig. 1. Phase diagrams at $g=0.5$ and fixed values $I_{\varepsilon}$ : (a) $I_{\varepsilon}=1.2$; (b) $I_{\varepsilon}=5$; (c) $I_{\varepsilon}=8$. Lines 1 and 2 define the boundary of stability domains of sliding (SF), dry (DF), and stick-slip (SS) friction ( $T$ is the tricritical point, $C$ is the critical point).

that this domain decreases with increase of the shear modulus value $G$ and decrease of the characteristic value of shear viscosity $\eta_{0}$.

The consideration of the additive noises of $\sigma, \varepsilon$, and $T$ shows that the stochasticity influence is non-essential for the shear component of elastic stress tensor and it is crucial for both the corresponding component of strain and the lubricant film temperature. The boundary of the domain of sliding friction is fixed by the equality for the noise intensities

$$
I_{T}=2 I_{\varepsilon}-\frac{1+g}{2 g^{2}},
$$

following from Eq. (14) at the conditions $x=1(\sigma=0)$ and $T_{e}=0$. According to Eq. (24) in absence of the temperature noise the lubricant melting occurs if the noise intensity of the shear strain component exceeds the value

$$
I_{\varepsilon 0}=\frac{1+g}{4 g^{2}},
$$

corresponding to the point $O$ in Fig. 2. The increase of both the shear strain and the temperature noises causes the lubricant melting if their intensities are bounded by condition (24). The domain of the stick-slip friction appears with further increase of these intensities above magnitudes

$$
I_{\varepsilon 1}=\frac{2+g}{2 g^{2}}, \quad I_{T 1}=\frac{3+g}{2 g^{2}}
$$




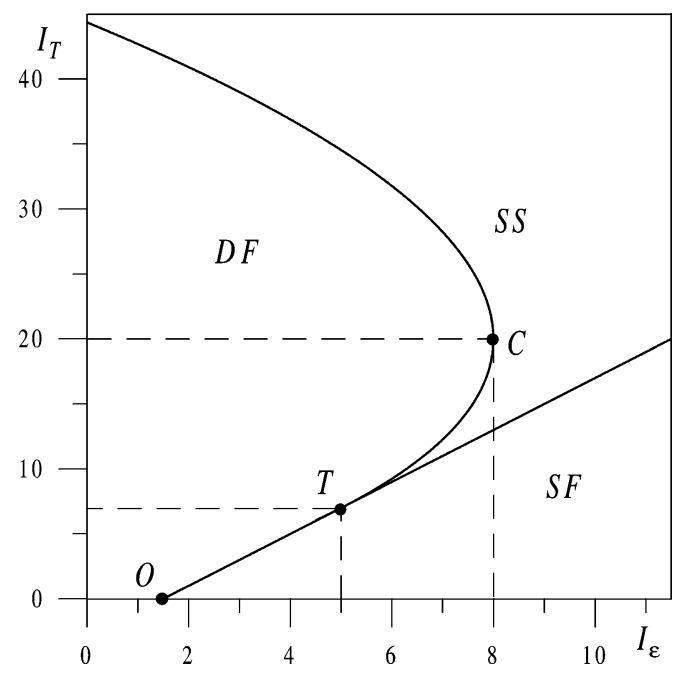

Fig. 2. Phase diagram for the system with $T_{e}=0, g=0.5$, and $I_{\varepsilon}, I_{T} \neq 0$.

at the tricritical point $T$ in Fig. 2. Such an intermittent behavior is realized within the region located above straight line (24) and outside the curve that is determined by

$$
I_{\varepsilon}=I_{T}\left[1+\frac{g}{3(1-g)}\right]+\frac{4 g}{27(1-g)^{2}}-\left\{\frac{2 g^{2}}{27(1-g)}\left[\frac{4}{3(1-g)^{2}}\left(\frac{2}{9(1-g)}+I_{T}\right)+\frac{2 I_{T}^{2}}{1-g}+I_{T}^{3}\right]\right\}^{1 / 2} .
$$

If the noise intensity of the shear strain exceeds the value $I_{\varepsilon 2}$ defined by (27) with the temperature noise $I_{T 2}=$ $2(3-g) / g^{2}$ (the point $C$ in Fig. 2), the dry friction region disappears at all. The curve (27) intersects the vertical axis at the point

$$
I_{T 3}=\frac{1}{2}\left\{\frac{27(1-g)}{2 g^{2}}+\frac{9}{g}-\frac{1}{2(1-g)}+\left[\left(\frac{27(1-g)}{2 g^{2}}+\frac{9}{g}-\frac{1}{2(1-g)}\right)^{2}+\frac{16}{g(1-g)}\right]^{1 / 2}\right\}
$$

above that the dry friction does not take place. The corresponding phase diagram depicted in Fig. 2 has a very non-trivial form (especially, within the domain $I_{\varepsilon 1} \leqslant I_{\varepsilon} \leqslant I_{\varepsilon 2}$ ).

\section{Summary}

The above consideration of the thermal and elastic fields noise influence on the solid-liquid transition of ultrathin lubricant film permits to define the domains of dry, sliding, and stick-slip friction modes in the phase diagram. So that, an evidence of the phase diagram complication is obtained due to studied fluctuations. Depending on the initial conditions the growth of lubricant film's temperature noise can decrease or increase friction, but the growth of elastic shear strain noise increases the sliding friction region only. It is shown that dry friction domain is bounded by relatively small values of the confining walls temperature and the noise intensities of lubricant strain and temperature (see Figs. 1 and 2). Thus, used here approach predicts the possibility for controlling of frictional behavior.

Above the concept of dynamical shear melting of the ultrathin lubricant film has been used [10,11]. In accordance with it the stick-slip friction can be described and such melting is represented as a result of action of elastic field of shear stress component caused by the heating of friction surfaces above the critical value. The essential limitation of this approach is the fact that stick-slip motion I studied was independent of the way in which the 
system was driven, i.e., elasticity and mass of the confining walls, although for such friction mode the mentioned dependence is crucial. However, it is worth noting that here the temperature of the confining walls $T_{e}$ plays a role of the parameter of external influence and the friction force is supposed to be decreasing with increasing velocity at the contact $V=l \partial \varepsilon / \partial t$.

\section{Acknowledgements}

This work is dedicated to memory of my friend and colleague Dr. E.A. Toropov. I am grateful to Prof. A.I. Olemskoi for helpful suggestions, Dr. I. Krakovsky and Dr. M. Marvan for fruitful discussions and hospitality during stay in Charles University, Prague, and I. Lyashenko for help with the numerical analysis of Eq. (14) and graphics. The work was partly supported by the grant of Ministers Cabinet of Ukraine.

\section{References}

[1] B.N.J. Persson, Sliding friction, in: Physical Principles and Applications, Springer-Verlag, Berlin, 1998.

[2] F. Family, Y. Braiman, H.G.E. Hentschel, in: D.E. Wolf, P. Grassberger (Eds.), Friction, Arching, Contact Dynamics, World Scientific, Singapore, 1996.

[3] Y. Braiman, H.G.E. Hentschel, F. Family, C. Mak, J. Krim, Phys. Rev. E 59 (1999) R4737.

[4] O.M. Braun, Yu.S. Kivshar, Phys. Rev. B 43 (1991) 1060.

[5] J.B. Sokoloff, Phys. Rev. B 51 (1995) 15573.

[6] T. Kawaguchi, H. Matsukawa, Phys. Rev. B 56 (1997) 4261.

[7] H. Yoshizawa, J. Israelachvili, J. Phys. Chem. 97 (1993) 11300.

[8] E.D. Smith, M.O. Robbins, M. Cieplak, Phys. Rev. B 54 (1996) 8252.

[9] J. Krim, D.H. Solina, R. Chiarello, Phys. Rev. Lett. 66 (1991) 181.

[10] J.M. Carlson, A.A. Batista, Phys. Rev. E 53 (1996) 4153.

[11] I.S. Aranson, L.S. Tsimring, V.M. Vinokur, Phys. Rev. B 65 (2002) 125402.

[12] S. Nasuno, A. Kudrolli, A. Bak, J.P. Gollub, Phys. Rev. E 58 (1998) 2161.

[13] L. Bocquet, W. Losert, D. Schalk, T.C. Lubensky, J.P. Gollub, Phys. Rev. E 65 (2002) 011307.

[14] I.S. Aranson, L.S. Tsimring, Phys. Rev. E 65 (2002) 061303.

[15] A.V. Khomenko, O.V. Yushchenko, Phys. Rev. E 68 (2003) 036110.

[16] F.R. Eirich (Ed.), Rheology, Academic Press, New York, 1960.

[17] H. Haken, Synergetics. An Introduction, in: Nonequilibrium Phase Transitions and Self-Organization in Physics, Chemistry, and Biology, third ed., Springer, Berlin, 1983.

[18] A.I. Olemskoi, A.V. Khomenko, Zh. Exp. Teor. Fiz. 83 (1996) 2144, JETP 83 (1996) 1180.

[19] H. Risken, The Fokker-Planck Equation, Springer, Berlin, 1989.

[20] A.I. Olemskoi, Sov. Phys. Usp. 41 (1998) 269. 